\title{
Die traditionelle Tierethik und ihre Kritik: der moralische Individualismus und die Grenzen und Vorzüge der Wittgenstein'schen Alternative
}

Susana Monsó, Andreas Aigner, Herwig Grimm

\section{Einleitung}

Die wegweisenden Bücher Animal Liberation von Peter Singer $\left({ }^{4} 2009\right)$ und The Case for Animal Rights von Tom Regan (2004) sind Klassiker der akademischen Tierethik. In der Debatte um moralische Ansprüche von Tieren und in den einschlägigen tierethischen Argumenten zahlreicher Autorinnen und Autoren finden sie ungebrochen Niederschlag. Ein wesentlicher Gedanke war und ist, dass Tiere moralischen Status besitzen und um ibrer selbst willen zu berücksichtigen sind und nicht etwa, weil dies im Interesse von Menschen ist (vgl. z. B. David DeGrazia 2002, 13-18). Auch in vielen Weiterentwicklungen auf dem Feld der Tierethik bleibt die grundlegende Argumentationsform, nach der auch Singer und Regan den moralischen Status von Tieren begründen, dominant. Ein Teil dieses Erfolges ist sicherlich auch der bestechenden Klarheit und Einfachheit dieser Argumente geschuldet, die Phillip McReynolds (2004) prägnant zusammenfasst: „[W] henever moral standing is extended to a new group, it is granted to the new group to the extent of and on the basis of their similarity to members of the old group“ (64, Hervorhebung im Original). Dabei stellen Singer wie Regan die Ähnlichkeiten bestimmter Tiere und Menschen in den Vordergrund, auf deren Basis sie gleiche Berücksichtigung relevant Ähnlicher fordern, was z. B. bei Singer in den Slogan All animals are equal mündet.

Wie dieses Argument der Ausweitung der moralischen Gemeinschaft vor dem Hintergrund eines Wittgenstein'schen Ansatzes zur Tierethik zu bewerten ist, wird im Folgenden behandelt. Alice Crary kritisiert in ihrem 2010 veröffentlichten Artikel Minding what already matters: A critique of moral individualism das skizzierte Argument, das auch heute noch als „orthodoxe" Position der Tierethik bezeichnet werden kann. Singer und Regan stehen paradigmatisch für dieselbe und Crary subsummiert sie trotz der Unterschiede unter dem Begriff des „moralischen Individualismus“, da sie einer gemeinsamen Logik folgen: Der moralische Individualismus (im Folgenden kurz MI) beschreibt grob gesagt die Ansicht, dass die moralische 
Berücksichtigungswürdigkeit verschiedener Wesen an deren jeweilige individuelle Charakteristika gebunden ist. James Rachels, selbst ein moralischer Individualist, formuliert eine präzise Definition des MI: Die Art und Weise, wie ein Individuum behandelt werden darf, kann nicht durch einen Verweis auf die Gruppenzugehörigkeit (z. B. Spezieszugehörigkeit) festgelegt werden, sondern durch die Berücksichtigung ihrer oder seiner besonderen individuellen Charakteristika (James Rachels 1999, 173). Einfach gesagt: Wenn ich wissen will, wie ich ein Tier behandeln soll, dann liegt die Antwort auf die Frage „,im Tier selbst“ ${ }^{1}{ }^{1}$ Crarys scharfe Kritik richtet sich nun auf zwei Punkte: Einerseits basiere der MI auf falschen Annahmen über die Grundlage unserer moralischen Beziehungen zu anderen Menschen und andererseits führe er zu einem verzerrten Verständnis über unsere moralischen Beziehungen zu Tieren (Alice Crary 2010, 20). Einige Jahre später relativiert Crary ihre ursprüngliche Kritik und nimmt sie zum Teil zurück: Sie verteidigt den Gedanken, dass der MI nicht gänzlich aufgegeben werden muss, sondern dass wir statt des „traditionellen $\mathrm{MI}^{\text {“ }}$ in der Tierethik einem „alternativen MI“ folgen sollten (Alice Crary 2018, 164). Im vorliegenden Artikel diskutieren wir die Grenzen und Vorzüge ihrer ursprünglichen Kritik des MI und zeigen zugleich die möglichen Gründe auf, die Crary zur Änderung ihrer Ansichten bewegten.

Crary folgt wie Cora Diamond einem Wittgenstein'schen Ansatz in der Tierethik ${ }^{2}$, der sich in den Grundannahmen deutlich vom Zugang moralischer Individualisten wie Singer und Regan unterscheidet (Todd May 2014). Ihre Kritik am MI, so möchten wir zeigen, hat unserer Ansicht nach allerdings nur begrenzte Relevanz, da sie nur bestimmte Formen des MI trifft, nicht aber den MI insgesamt. Darüber hinaus, so ein vorweggenommenes Ergebnis unserer Analyse, ist der MI in bestimmter Hinsicht nicht überwindbar - ein Umstand, der für die Angemessenheit von Crarys Schritt hin zu einer qualifizierten Version des MI spricht. Wir wollen aber auch zeigen, dass die ursprüngliche Kritik immer noch aktuell ist und wichtige Einsichten liefert. Diese Punkte werden wir Schritt für Schritt erarbeiten. In Abschnitt 2 skizzieren wir die Wittgenstein'sche Kritik des MI.

1 Die hier verwendeten Bezeichnungen „traditionelle Tierethik“ und „Orthodoxie“ beziehen sich einerseits auf Singer und Regan, andererseits beschreiben sie auch Weiterentwicklungen dieser Arbeiten innerhalb des MI. Genannt seien etwa Gary Francione (1995), Bernard Rollin (2017), James Rachels (1999), Jeff McMahan (2005), Gary E. Varner (2012), Evelyn B. Pluhar (1995) und David DeGrazia (1996).

2 Ein anderer prominenter Autor, der Wittgensteins Ideen auf tierethische Fragen angewendet hat, hier aber nicht kommentiert wird, ist Raimond Gaita (2005). 
Abschnitt 3 erläutert die Grenzen dieser Kritik. In Abschnitt 4 stellen wir dessen Vorzüge dar. Abschnitt 5 widmet sich schließlich der Darstellung der häufigsten Einwände gegen den Wittgenstein'schen Zugang sowie der Frage, ob dieser Zugang tatsächlich als Alternative zur orthodoxen Position zu verstehen ist. Ziel ist es nicht, Crarys oder Diamonds Theorien zu verteidigen, sondern die Stärken und Schwächen ihrer Kritik an der traditionellen Tierethik zu analysieren und klarzustellen, welche Aspekte derselben immer noch von Relevanz sind bzw. einer Modifikation bedürfen oder verworfen werden sollten.

\section{Die Wittgenstein'sche Kritik am MI}

Crarys (2010) Kritik am MI ist eine Weiterentwicklung der Ideen, die bereits früher von Diamond in ihrer Kritik an den orthodoxen Zugängen zur Tierethik formuliert wurden. In ihrem 1978 erschienenen Artikel Eating Meat and Eating People (Cora Diamond 1978) zielt sie auf die gleichen Punkte ab, die Crary später als die zentralen Merkmale des MI beschreibt. Trotz unserer Fokussierung auf Crary werden wir daher zur besseren Veranschaulichung ihrer Ideen auch auf Diamond zurückgreifen, die wie Crary ihre Argumente in weiten Teilen auf Arbeiten von Ludwig Wittgenstein stützt. Obwohl in der Fachwelt darüber debattiert wird, worin Wittgensteins Beitrag zur Moralphilosophie überhaupt liegt, so scheint zumindest darin Einigkeit zu bestehen, dass er Reduktionismus in moralphilosophischen und anderen Fragen vermeidet (Benjamin De Mesel/James Thompson 2015, 1). Diamond (vgl. 1991 b, 21-23) orientiert sich in ihrem Zugang zur Moralphilosophie z. B. insofern an Wittgenstein, als sie keine allgemeinen normativen Prinzipien aufstellt, sondern die Aufmerksamkeit auf die gelebte Moral der Menschen (das „Tun“) und die vielfältigen Arten des moralischen Denkens lenkt.

Diamond und Crary kritisieren die traditionelle Tierethik aufgrund der dort vertretenen Ansicht, dass das bloße Menschsein alleine nicht ethisch relevant sei, sondern im Gegenteil die Zugehörigkeit zur Gruppe der Menschen eben keinen Grund für die Aufnahme in die moralische Gemeinschaft darstellt. Eines der bekanntesten Argumente in der Tierethik, das Singer bereits in Animal Liberation formulierte und das seither auf breite Resonanz gestoßen ist, vermittelt genau diese Idee: das Argument der marginal cases. Dem Argument der "Grenzfälle“ zufolge, das man auch unter dem argument from species overlap kennt (Oscar Horta 2014), gibt es keine besondere Fähigkeit, die erstens alle Menschen haben würden, zweitens allen anderen (nicht-menschlichen) Tieren fehlen würde und sich drittens 
eignen würde zu begründen, Wesen in die moralische Gemeinschaft aufzunehmen. Die mutmaßlich exklusiv menschlichen Fähigkeiten, die uns angeblich von Tieren unterscheiden (etwa Sprache, Intelligenz, die Fähigkeit, moralisch zu handeln etc.), werden in Wirklichkeit nicht von allen Menschen geteilt und kommen sogar bestimmten Tieren zu. Das Fehlen bzw. Vorhandensein bestimmter Fähigkeiten bei tierlichen wie menschlichen Grenzfällen weicht die klare Grenzziehung auf. Daher kann der Verweis auf Fähigkeiten auch nicht die Annahme begründen, dass alle und insbesondere nur Menschen moralisch zu berücksichtigen wären. Wer alle Menschen in der moralischen Gemeinschaft halten will, muss weniger anspruchsvolle Fähigkeiten wählen, die aber bestimmte Menschen und bestimmte Tiere teilen. Wird die moralische Berücksichtigungswürdigkeit eines Lebewesens aber nicht anhand der erwähnten, anspruchsvollen Eigenschaften bestimmt, sondern stattdessen z. B. anhand der Empfindungsfähigkeit (Sentientismus), erweitert sich der Kreis der moralischen Gemeinschaft. Dieses Argument funktioniert allerdings nur, wenn man davon ausgeht, dass die eine Eigenschaft, die allen Menschen gemeinsam ist, nämlich ihre Zugehörigkeit zur Spezies Mensch, nicht als Grund für eine Vorzugsbehandlung herangezogen werden kann. Da die schiere Zugehörigkeit zu einer Spezies moralisch irrelevant ist, besteht der Anspruch des Arguments der Grenzfälle zugleich darin, den Speziesismus zu überwinden. In Analogie zum Rassismus und Sexismus bestimmt Richard Ryder (1971) den Speziesismus als eine illegitime Bevorzugung von Vertretern einer bestimmten (meist der eigenen) Spezies und Abwertung aller anderen allein aufgrund der Spezieszugehörigkeit.

Crary und Diamond stehen diesem Argument sehr kritisch gegenüber, und zwar nicht, weil sie die Notwendigkeit abstreiten würden, Tiere vor unethischer Behandlung zu bewahren. Stattdessen konstatieren sie, dass dieses Argument sowohl unser Verständnis von moralischen Beziehungen zu anderen Menschen verzerrt - weil diese Beziehungen nicht auf „kühlen“ Kalkulationen hinsichtlich des Besitzes bestimmter Fähigkeiten und ihrer moralischen Relevanz basieren - als auch wenig überzeugende Gründe für die ethische Behandlung von Tieren liefert. Darüber hinaus ergibt sich aus ihrer Perspektive die erschreckende Vorstellung, dass man auf das Argument der Grenzfälle nicht nur mit einer besseren Behandlung von Tieren reagieren könnte, sondern mit einer schlechteren Behandlung von menschlichen „Grenzfällen“. Crary und Diamond argumentieren ferner, dass die Orthodoxie in der Tierethik die Funktionsweise unserer gelebten Moral missversteht. Denn die Art und Weise, wie wir z. B. beeinträchtigte Menschen behandeln, zeigt, dass „verminderte“ Fähigkeiten nicht generell als Basis zur Rechtfertigung einer schlechteren Behandlung herangezogen 
werden. Vielmehr begründet das Fehlen bestimmter Fähigkeiten (z. B. bei einer Person mit kognitiver Beeinträchtigung) spezielle moralische Rücksichtnahme (Alice Crary 2010, 21).

Ein weiteres Beispiel für Crarys und Diamonds Kritik bezieht sich auf die Annahme, dass die traditionelle Tierethik verkennt, wie menschliche Gesellschaften ihre Toten behandeln. Toten Menschen fehlt es nicht nur an kognitiven Fähigkeiten, sondern auch an jeder Form von Subjektivität. Dennoch werden die Körper von Verstorbenen mit Respekt behandelt: Wir sind davon überzeugt, dass diese nicht verunstaltet, verstümmelt, entwürdigend zur Schau gestellt oder gar verspeist werden sollten. Das Argument der Grenzfälle, so Crary und Diamond, könne nicht einmal ansatzweise erklären, warum wir uns z. B. eher vorstellen können, eine Kuh zu essen, die vom Blitz getroffen wurde, als einen Menschen, dem das gleiche Schicksal zuteilwurde (Cora Diamond 1978, 468).

Doch für Crary und Diamond zeigen Praktiken wie die grundsätzliche Weigerung, tote Menschen zu essen, worin das Menschsein besteht. Es geht hier weder darum, dass es „moralisch falsch“ ist, Menschen zu essen, noch darum, dass Menschen dies ablehnen, weil sie dadurch jemandes Interessen respektieren; stattdessen wird dadurch verdeutlicht, dass Praktiken wie diese zur Bestimmung des Begriffs „Mensch“ beitragen (Cora Diamond 1978, 469-470). Wie Diamond sagt, lernen wir am Esstisch, dass Menschen manche Tiere essen, nicht aber andere Menschen (was allerdings nicht impliziert, dass Diamond den Fleischkonsum verteidigt oder Fleischkonsum moralisch richtig ist). Mensch zu sein bedeutet unter anderem, dass man nicht gegessen wird. Da Singer und Regan diese Tatsache ignorieren, entsprechen ihre Argumente weniger einem Plädoyer für die moralische Berücksichtigung von Tieren, sondern vielmehr einem Angriff auf etwas im menschlichen Leben Bedeutsames (Cora Diamond 1978, 471). In der Wittgenstein'schen Perspektive geht es bei der Verteidigung von Tieren darum, die Bedeutsamkeit dessen zu berücksichtigen, wie Menschen über Tiere denken und sprechen und wie wir die Welt und uns selbst verstehen. Dies erreichen wir nicht durch eine bloße Reflexion über biologische Fakten im Hinblick auf tierliche Eigenschaften, sondern über die Beleuchtung unseres Sprachgebrauchs, von künstlerischen Bestrebungen und Narrativen, die unsere moralische Vorstellungskraft prägen und anregen. In „methodologischer" Hinsicht impliziert dies z. B. den Einsatz von Literatur, um unsere Vorstellungskraft auch in moralischen Fragen anzuregen, und nicht die Suche nach moralisch relevanten biologischen Fakten.

Crary und Diamond sehen in der Tendenz orthodoxer tierethischer Autorinnen und Autoren, die Welt zu beschreiben als sei sie frei von Werten, den entscheidenden Faktor hinter ihrer wenig ansprechenden Darstellung 
der Moral an. Genau das ist es, was Crary mit dem MI identifiziert. Als ein Prinzip, das die moralische Berücksichtigung anderer anhand ihrer individuellen Charakteristika begründet, will uns der MI dazu bewegen, so „objektiv" wie möglich auf die Welt zu blicken. Da Tatsachen als Grundlage für Fragen der Moral angesehen werden, ist es entscheidend, sie klar zu sehen. Dies bringt den MI in eine Nahebeziehung zum naturwissenschaftlichen Weltbild, was sich in der Idee einer beobachter-unabhängigen Welt widerspiegelt, die objektiv beschrieben werden kann. ${ }^{3}$ Gleichzeitig wird die Welt in dem Sinne als neutral angesehen, als sie der Ethik unabhängig unserer moralischen Fähigkeiten gegeben ist (Alice Crary 2010, 41). Für Crary und Diamond besteht das Problem darin, dass die Konzepte von „Mensch“ und „Tier“ aber keine rein biologischen sind. Diese Konzepte beziehen sich zwar auf Wesen mit bestimmten biologischen Charakteristi$\mathrm{ka}$, aber wenn wir dabei nur an eine biologische Bedeutung denken, lassen wir viel außer Acht, das entscheidend für unsere gelebte Moral ist.

\section{Grenzen der Wittgenstein'schen Kritik}

Ein guter Ausgangspunkt, um die Grenzen der Wittgenstein'schen Kritik am MI deutlich zu machen, ist der Rekurs auf die erste explizite Definition des MI von James Rachels: „The basic idea is that how an individual may be treated is to be determined, not by considering his group memberships, but by considering his own particular characteristics" $(1999,173)$. Obwohl sich Crary explizit auf Rachels bezieht, führt sie die Idee des MI in zwei Hinsichten enger, wenn sie schreibt:

„What distinguishes approaches in ethics that count as forms of moral individualism is the claim that a human or nonhuman creature calls for specific forms of treatment only insofar as it has individual capacities such as, for instance, the capacity for suffering or the capacity to direct its own life" (Alice Crary 2010, 20, Hervorhebung im Original).

Der erste Unterschied zwischen Rachels' ursprünglicher Definition und Crarys neuer Formulierung besteht darin, dass dort, wo Rachels von Eigenschaften oder Charakteristika (engl. characteristics) spricht, Crary von Fähigkeiten (engl. capacities) spricht. Das ist keine unwesentliche Änderung Crarys, da der Begriffsumfang von „Eigenschaft“ viel weiter ist als je-

3 Ein Argument dazu, wie „Objektivität“ in die Wittgensteinianische Ethik integriert werden kann, wurde von Benjamin De Mesel (2018, 91-110) formuliert. 
ner der „Fähigkeit“. Alle Fähigkeiten eines Individuums sind zugleich Eigenschaften bzw. Charakteristika, aber es handelt sich nicht bei jeder Eigenschaft eines Individuums zugleich um eine Fähigkeit. Entsprechend umfasst ein auf Merkmale bezogener MI prima facie wesentlich mehr Theorien als ein auf Fähigkeiten bezogener MI.

Crarys Reduzierung des MI auf seine fähigkeitsbasierte Form ist kein Einzelfall innerhalb metaethischer Überlegungen in Bezug auf die Tierethik. Sie zeigt sich z. B. auch in Todd Mays (2014) Unterscheidung zwischen MI und moralischem Relationalismus (engl. moral relationalism). May definiert den moralischen Relationalismus als jene Position, die den moralischen Status eines Lebewesens primär aus ihrer oder seiner Beziehung zu Menschen begründet und nicht nur aus dessen besonderen Charakteristika (2014, 155). Er stellt den moralischen Relationalismus dem MI gegenüber, der seiner Ansicht nach die moralische Berücksichtigungswürdigkeit eines Individuums durch einen Verweis auf Fähigkeiten begründet (engl. capacity-based reasons), nicht durch einen Verweis auf relationale Gründe (engl. relation-based reasons; Todd May 2014). Diese Unterscheidung ist allerdings irreführend, denn schon alleine die Tatsache, dass Beziehungen auch Eigenschaften bzw. Charakteristika von Individuen sind, wirft die Frage auf, warum der moralische Relationalismus nicht als eine Form von MI betrachtet werden sollte. Da sowohl Fähigkeiten als auch Beziehungen Eigenschaften von Individuen sind, kann der MI, so wie er von Rachels ursprünglich definiert wird, integrativ verstanden werden: Er inkludiert einerseits Zugänge, die sich auf intrinsische Eigenschaften bzw. Fähigkeiten wie Empfindungsfähigkeit und Bewusstsein (vgl. Nicolas Delon 2015) berufen, um die moralische Berücksichtigungswürdigkeit eines Lebewesens zu begründen (z. B. Singers Präferenz-Utilitarismus, Regans Rechteansatz oder Martha C. Nussbaums [2007] Fähigkeiten-Ansatz), und andererseits Zugänge, die zusätzlich Kontexte und Beziehungen hervorheben (z. B. Clare Palmers [2010] kontextualistischer Ansatz).

Crarys Gleichsetzung des MI mit einem auf Fähigkeiten basierten MI wird unserer Ansicht nach zum Kernproblem ihrer Kritik. Dazu gleich mehr, doch zunächst wollen wir noch einen zweiten Unterschied zwischen Rachels' und Crarys Definition des MI hervorheben. In Rachels' ursprünglicher Formulierung wird der MI als metaethisches Prinzip betrachtet, das sich auf die Frage bezieht, wie man Unterschiede in der Behandlung von Individuen rechtfertigt. Der Grundgedanke liegt darin, dass man zwei Individuen nicht unterschiedlich behandeln darf, wenn kein moralisch relevanter Unterschied angegeben werden kann, der diese Ungleichbehandlung rechtfertigt. Rachels $(1999,177)$ nennt als Beispiel einen Arzt, der zwei Patientinnen oder Patienten hat und vor der Wahl der jeweils 
passenden Behandlung steht. Während er der Person mit der Infektion Penizillin verabreicht, legt er der Person mit dem gebrochenen Arm einen Gips an. In diesem Fall wäre die Ungleichbehandlung gerechtfertigt, weil es einen relevanten Unterschied zwischen den beiden Personen gibt. Der MI ist daher als Gleichheitsprinzip gedacht, das die gleiche Berücksichtigung von Gleichen (bzw. gleichen Interessen) garantiert.

Im Unterschied dazu beschreibt Crary den MI als Argument über den moralischen Status: Ob und in welchem Ausmaß ein Wesen einen moralischen Status besitzt, leite sich ihr zufolge beim MI aus dessen individuellen Fähigkeiten ab (Alice Crary 2018, 158). Obwohl dies in ihrer ursprünglichen Definition nicht so klar ersichtlich ist, zeigt es sich aber immer wieder in ihren Arbeiten. So schreibt sie etwa, dass der MI davon ausgehe, dass nur intrinsische Eigenschaften (engl. intrinsic characteristics) einem Wesen moralischen Status verleihen (Alice Crary 2010, 21), und dass der MI behaupte, dass nur tierliche Individuen mit bestimmten geistigen Fähigkeiten (engl. mental capacities) moralische Berücksichtigung verdienen (Alice Crary 2010, 41). Der MI sei eine Doktrin, welche die Antwort auf die Frage, ob ein Wesen einen moralischen Status besitzt, an dessen individuelle Fähigkeiten knüpfe (Alice Crary 2018, 158). Indem Crary den MI speziell als Doktrin über den moralischen Status auffasst und nicht generell als Prinzip hinter ethischen Urteilen, exkludiert sie alle Ansätze, die nicht auf die Begründung des moralischen Status zielen, wie etwa die Tugendethik (z. B. Rosalind Hursthouse 2011). Es ist aber schwerlich einzusehen, weshalb Tugendethikerinnen und Tugendethiker nicht für die gleiche Berücksichtigung Gleicher stehen sollten, schon alleine aufgrund der Tatsache, dass sich das Gleichheitsprinzip prominent bei Aristoteles findet.

Dies bedeutet jedoch nicht, dass Rachels' ursprüngliche Definition fraglos akzeptiert werden sollte. So zeigt sich bei genauerem Hinsehen zumindest folgendes Problem: Ihm zufolge darf die Gruppenzugehörigkeit nicht das ausschlaggebende Kriterium für die Festlegung der Art der Behandlung eines Individuums sein; stattdessen müssen dessen individuelle Charakteristika berücksichtigt werden (James Rachels 1999, 173). Was hier ins Auge sticht, ist die Tatsache, dass Gruppenzugehörigkeiten ja selbst zu den Charakteristika eines Individuums gehören. Dieser augenscheinliche Widerspruch könnte durch die Behauptung aufgelöst werden, der MI schließe Gruppenzugehörigkeiten aus den moralisch relevanten Charakteristika eines Individuums aus. Dies würde den MI jedoch als absurdes Prinzip erscheinen lassen, da es durchaus einige Gruppenzugehörigkeiten gibt, die zur Bestimmung dessen herangezogen werden können, wie ein Individuum zu behandeln ist. Als Beispiel sei hier ein Trainer genannt, der nur Spielerinnen und Spielern seines Fußballteams den Zutritt auf den Sport- 
platz während des Trainings erlaubt, während er anderen Personen den Zutritt verweigert. Obwohl der Trainer verschiedene Individuen aufgrund ihrer Gruppenzugehörigkeit unterschiedlich behandelt, verletzt er hier nicht das Gleichheitsprinzip. Wir würden es wahrscheinlich sogar als seltsam ansehen, fühlte sich hier eine zufällige, nicht zum Team gehörige Person ungerecht behandelt, nur weil sie vom Spielfeld verwiesen wird. Gerade weil diese Person nicht zur Gruppe „Fußballteam“ gehört, ist deren Ausschluss vom Training legitim, und zwar gleichgültig, ob sie in guter körperlicher Verfassung und für den Sport geeignet wäre. Mitglied einer bestimmten Gruppe zu sein ist ein Kriterium, um aus Gleichen Ungleiche zu machen und eine unterschiedliche Behandlung zu rechtfertigen. Daher können Gruppenzugehörigkeiten - auch wenn sie als extrinsische und nicht als intrinsische Eigenschaften angesehen werden können - nicht von der Liste jener Charakteristika ausgeschlossen werden, die bestimmen, wie ein Individuum behandelt werden sollte.

Rachels' Definition zielt jedoch in der Bestimmung dessen, was moralisch irrelevant ist, anscheinend nicht generell auf Gruppenzugehörigkeit ab, sondern speziell auf Spezieszugehörigkeit (1999, 173-174). Rachels' Definition des MI ist daher auf die Überwindung des Speziesismus ausgerichtet, also die Überwindung der unterschiedlichen Behandlung von Individuen nur aufgrund der bloßen Spezieszugehörigkeit. Der Grund, warum der Speziesismus seit seiner ersten Formulierung als Diskriminierung angesehen wird, liegt jedoch nicht darin, dass die Zugehörigkeit zu einer Spezies auf die Zugehörigkeit zu einer Gruppe hinausläuft, sondern weil Spezieszugehörigkeit als moralisch irrelevantes Charakteristikum eines Individuums gilt. Werden zwei Individuen unter den gleichen Umständen unterschiedlich behandelt, muss eine Eigenschaft aufgezeigt werden können, die dies legitimiert. Diese Idee spiegelt sich in Rachels' Formulierung des Gleichheitsprinzips wider: „Individuals are to be treated in the same way unless there is a relevant difference between them that justifies a difference in treatment" (1999, 176, unsere Hervorhebung). Obwohl das Menschsein in manchen Weltanschauungen als moralisch relevantes Merkmal angesehen wird, z. B. aufgrund der Annahme, dass Menschen als Ebenbilder Gottes geschaffen wurden, so hat die Auffassung, dass die schiere Spezieszugehörigkeit einen relevanten Unterschied in der Moral machen würde, einen schlechten Stand.

Auch wenn das Gleichheitsprinzip oft gegen den Speziesismus angeführt wird, ist es - wie auch der MI selbst - im Hinblick auf den normativen Rahmen und das zugrunde liegende Wertesystem neutral. Um dies zu präzisieren: Keines der beiden Prinzipien legt fest, wie Individuen zu behandeln sind oder was als ein moralisch relevanter Unterschied zählt. Des- 
halb folgt die Ablehnung des Speziesismus auch nicht automatisch aus dem Gleichheitsprinzip, und deshalb ist auch Crarys Kritik daran, dass der MI die moralische Relevanz des Menschseins bestreitet, irreführend. Ein Mensch zu sein ist nämlich ein Charakteristikum, das auf Individuen zutrifft, daher würde eine Theorie, die aus dem Menschsein bestimmte moralische Ansprüche ableitet, ebenso zum MI zählen können. Obwohl Vertreterinnen und Vertreter des MI das Menschsein traditionell als moralisch irrelevant ansehen (weil sie es als rein biologische Kategorie verstehen), ist dies kein notwendiger Wesenszug des MI, sondern hängt vielmehr von den jeweiligen Kriterien ab, die jemand für moralische Relevanz anführt.

Crarys und Diamonds Argumente laufen im Wesentlichen auf eine Verteidigung der Idee hinaus, dass das Menschsein moralisch relevant ist, weil sie die moralische Relevanz als etwas verstehen, das de facto in einem deskriptiven Sinne moralisch relevant ist. Die Wittgenstein'sche Kritik richtet sich daher nicht gegen die Grundidee des MI, sondern auf den Ausschluss des Menschseins als moralisch relevantes Charakteristikum innerhalb der traditionellen Tierethik. Aus Crarys und Diamonds Sicht entspricht dies nicht unserer gelebten Moral, was sie als entscheidenden Fehler der traditionellen Tierethik ansehen. Indem man jedoch einräumt, dass auch das Menschsein ein Charakteristikum von Individuen ist, erweist sich die Annahme des Wittgenstein'schen Zugangs über die moralische Bedeutsamkeit des bloßen Menschseins als durchaus kompatibel mit dem MI.

Crary und Diamond problematisieren zudem die Tendenz des traditionellen MI, die moralisch relevanten Merkmale von Individuen auf eine einzelne Fähigkeit oder ein bestimmtes Set an festgelegten Fähigkeiten zu reduzieren. Dies bringt ihnen zufolge die - bereits erwähnte - fragwürdige Implikation mit sich, dass tote Menschen oder Menschen mit einer schweren Beeinträchtigung bzw. Behinderung keine oder weniger moralische Berücksichtigung verdienen, weil sie nicht oder nur in reduziertem Ausmaß über die nötigen Fähigkeiten für moralische Rücksichtnahme verfügen. Obwohl diese Kritik sicherlich auf manche Zugänge zum MI zutrifft (z. B. auf Singers oder McMahans Utilitarismus), so möchten wir betonen, dass dies jedoch ein kontingentes Charakteristikum ist, das nur für bestimmte Formen des MI gilt. Eine normative Theorie, die auf Basis ihrer axiologischen Grundlage nur subjektiv erfahrene Formen von Schaden als moralisches Problem anerkennt (wie der Hedonismus), kann nicht erfassen, inwiefern Leichname respektlos behandelt werden können oder inwiefern verschiedene Formen von Schaden auch Menschen mit Beeinträchtigung betreffen, die den Schaden gar nicht subjektiv als solchen wahrnehmen. Wir halten dies für eine Schwachstelle des hedonistischen und des Präferenz-Utilitarismus. Dies gilt jedoch auch für andere Theorien 
mit entsprechenden axiologischen Grundlagen, aber diese Schwachstelle rührt eben vom jeweiligen Wertesystem her und nicht von ihrem moralisch-individualistischen Charakter.

Der MI ist vom Prinzip her gut mit einem Wertesystem vereinbar, das von objektiven Werten und objektivem Schaden ausgeht, da der MI, wie bereits erwähnt, als ein axiologisch-neutrales Prinzip angesehen werden kann. Darüber hinaus besitzen auch Crary und Diamond anscheinend einen individualistischen Zugang zur Antwort auf die Frage, wie man Pflichten gegenüber beeinträchtigten und toten Menschen begründet. Denn diese Pflichten beziehen sich auf die individuellen Charakteristika eines Wesens. Diamond sagt hierzu Folgendes:

„It would be held by many people uninfluenced by theories of what can count as moral relevance that the conviction by a court of a severely retarded $[s i c]$ person for a crime that required an intention the retarded person could not form was unjust; the less capable of forming such an intention the person is, the more palpable the injustice" (Cora Diamond 1991 a, 53).

Wird eine Person mit schwerer kognitiver Beeinträchtigung für ein Verbrechen verurteilt, das z. B. eine Absicht oder zielgerichtete Planung voraussetzt, zu der diese Person gar nicht in der Lage war, sind es genau diese individuellen Charakteristika, die die Verurteilung so ungerecht erscheinen lassen. Die Annahme, dass der verurteilten Person ein Schaden zugefügt wird, gerade weil sie eine Beeinträchtigung oder Behinderung hat, erweist sich daher als das Resultat einer individualistischen Perspektive. Dementsprechend ist der MI auch mit Crarys Konzeptualisierung geistig schwer beeinträchtigter Menschen vereinbar: Diese Menschen „[have] suffered from a great deprivation in virtue of which they merit special solicitude“ (Alice Crary 2010, 33). Ihr zufolge sind es genau diese schweren Beeinträchtigungen eines Menschen, die andere Menschen auf den Gedanken bringen, dass sie eine spezielle Fürsorge verdienen (Alice Crary 2010, 21, 33). Dabei handelt es sich um eine Beschreibung, die auf die besonderen Charakteristika eines Individuums eingeht und die so aufgefasst werden kann, dass diese Charakteristika spezielle Ansprüche erzeugen. In ähnlicher Weise kann der MI auch erklären, warum es, wie Crary (2010) aufzeigt, besonders verwerflich ist, z. B. einen Menschen mit Alzheimer-Demenz zu übervorteilen bzw. auszunutzen (22). Es ist deshalb so verwerflich, weil diese Person durch ihre besonderen Charakteristika in Zusammenhang mit der Erkrankung eine erhöhte Vulnerabilität besitzt.

Darüber hinaus kann der MI zur Erklärung dessen herangezogen werden, warum wir toten Menschen eine besondere Behandlung schulden. 
Hierbei geht es nicht um das Kriterium der Subjektivität bzw. des subjektiv Empfundenen, aber die Körper der Toten können dennoch auf eine bestimmte individualistische Weise beschrieben werden. Crary spricht etwa von "fellows who have reached the end of their mortal threads" $(2010,34)$. Solch eine individualistische Beschreibung eines toten Mitmenschen kann, abhängig von unserer jeweiligen normativen Theorie, bestimmte Formen von Respekt begründen, die über die Maximierung von Lust oder das Kalkulieren von Präferenzen hinausgehen.

Der MI kann daher die Existenz von Pflichten gegenüber toten Menschen und Menschen mit Beeinträchtigung bzw. Behinderung ebenso gut erklären wie die Pflichten gegenüber lebenden und nicht beeinträchtigten Menschen, zumindest solange wir dazu bereit sind, über hedonistische und präferenzbezogene Werttheorien hinauszugehen. Allerdings gibt es noch einen weiteren Aspekt, in dem die Wittgenstein'sche Kritik am MI an der Sache vorbeigeht. Dieser betrifft den Punkt, dass eine gewisse Form von Individualismus unvermeidlich ist. Denn ungeachtet des normativen Rahmens und der axiologischen Grundlage, von dem bzw. der wir ausgehen, und ungeachtet unserer Auffassungen in Zusammenhang mit Moralpsychologie und Metaphysik, sobald wir mit ethischen Fragen beschäftigt sind, müssen wir unweigerlich ein bestimmtes Maß an Aufmerksamkeit auf das Individuum richten, das aktuell oder potentiell von unseren Handlungen betroffen ist. Wir mögen vielleicht Spezieszugehörigkeit als moralisch relevant oder nicht relevant betrachten und wir mögen vielleicht den moralischen Status als nützlichen oder unnützen Begriff ansehen, aber die besonderen Charakteristika jener Individuen, mit denen wir konfrontiert sind, geben stets relevante Informationen - und sei es nur, um festzustellen, ob es sich um ein Lebewesen oder ein unbelebtes Objekt handelt, ob es ein Gegenstand, ein Leichnam oder etwas anders ist. Dies lässt sich leicht aufzeigen, indem man über utilitaristische oder tierrechtliche Zugänge zur Tierethik hinausblickt. Gemäß der Tugendethik sind etwa die Merkmale der konkreten Situation, in der man sich befindet, einschließlich der Charakteristika der Individuen, mit denen man konfrontiert ist (z. B. „Welcher Spezies gehört dieses Individuum an?"“, „In welcher Situation befindet sich das Individuum?“ oder „In welcher Beziehung steht das Individuum zu mir?"), für die Bestimmung dessen wichtig, was in dieser Situation tugendhaft wäre (vgl. z. B. Rosalind Hursthouse 2006). So kommt auch Nussbaums (2007) Fähigkeiten-Ansatz nicht umhin, Bezug darauf zu nehmen, um wen oder was es sich bei einem Individuum handelt. Denn je nachdem, um welche Art Individuum es sich handelt, entscheidet darüber, was zum Gedeihen des Individuums beitragen kann und wodurch es geschädigt werden kann. 
Die Unvermeidlichkeit des MI dürfte ein entscheidender Grund dafür sein, dass Crary ihre ursprüngliche Kritik teilweise zurücknimmt. Sie schreibt in einem aktuelleren Artikel zum Thema unter Bezugnahme auf ihre bevorzugten Zugänge zur Tierethik:

„[T]here are other thinkers who take up questions of animals and ethics and who, while differing substantially from Singer et al. in their views, nevertheless resemble them in basing their conclusions about moral standing on attention to individual creatures. In light of this convergence, it makes sense to withhold the generic label ,moral individualism' from the projects of Singer and likeminded others and to place their work instead under the heading of traditional moral individualism" (Alice Crary 2018, 158).

Im Zuge ihrer Fürsprache für diesen alternativen Zugang zur Tierethik schreibt Crary über einen auch von Lori Gruen (2014) geschilderten Fall eines Schimpansen, der in der Unterhaltungsindustrie aufgewachsen ist und darauf trainiert wurde, in Gegenwart von Menschen ein vermeintlich lustiges Gesicht zu ziehen. Obwohl der Schimpanse in der Zwischenzeit geschützt lebt und nicht mehr zur Unterhaltung eingesetzt wird, zieht er immer noch das gleiche Gesicht, wenn er auf Menschen trifft. Indem der Schimpanse auf ein Verhalten konditioniert wurde, das Gruen als für ihn entwürdigend erachtet, so Crary (2018), hat man dieses Individuum auf eine Weise geschädigt, die der Utilitarismus nicht zu erfassen vermag, weil der Schimpanse den Schaden nicht thematisieren kann. Um den Schaden zu erkennen, müsse unsere Aufmerksamkeit auf das Individuum selbst gerichtet werden (Alice Crary 2018, 164). Die Tatsache, dass dieses Individuum ein Schimpanse ist, ein majestätisches Geschöpf, dem wir in der Wildnis wohl mit Furcht begegnen würden, und die Tatsache, dass er als dieser Schimpanse eine besondere Geschichte hat, lässt uns die immer noch sichtbaren Zeugnisse seines früheren Trainings als Verletzung seiner Würde ansehen. So räumt Crary (2018) letztlich ein, dass eine gewisse Aufmerksamkeit für das Individuum unvermeidlich ist, und sie revidiert ihre ursprüngliche Forderung, den MI gänzlich aufzugeben. Stattdessen spricht sie von der Notwendigkeit einer nicht-traditionellen, alternativen Form des MI (Alice Crary 2018, 164).

\section{Vorzüge der Wittgenstein'schen Kritik}

Die Probleme, die Diamond bereits 1978 thematisiert und Crary später aufgreift, rühren nicht so sehr von der Tatsache her, dass traditionelle $\mathrm{Zu}$ - 
gänge zur Tierethik individualistisch sind, sondern vielmehr daher, dass diese Zugänge folgende drei Merkmale aufweisen: 1. Rationalismus; 2. Naturalismus; 3. Reduktionismus. Diese drei Merkmale hängen zusammen und treffen nicht zwangsläufig auf jede Form des MI zu. Sie ergeben sich aus dem Fokus auf „nüchterne“ ethische Argumente, die aus dem Besitz bestimmter Charakteristika aufgebaut werden. Im Folgenden erläutern wir diese Merkmale und ihre Wittgenstein'sche Kritik.

\subsection{Kritik am Rationalismus}

Der traditionelle MI ist in einem spezifischen Sinn rationalistisch, weil er Emotionen gezielt aus der Diskussion über unsere Pflichten gegenüber Tieren auszuschließen versucht. Im Vorwort zur ersten Ausgabe von Animal Liberation aus dem Jahre 1975 beschreibt Singer, dass er das Buch nicht aufgrund seiner Tierliebe verfasst hat, sondern weil er die Frage, wie wir Tiere behandeln sollen, auf möglichst konsistente Weise durchdenken wollte ( ${ }^{4} 2009,10$, Anhang). Wiewohl er einräumt, dass seine Beschreibungen über Massentierhaltung und Tierversuche bestimmte Emotionen in den Leserinnen und Lesern auslösen könnten, sei die Begründung, die er für die Ablehnung dieser Praktiken gibt, eine Frage der Vernunft und allgemein akzeptierter moralischer Prinzipien - und nicht von Emotionen (Peter Singer ${ }^{4}$ 2009, 11, Anhang). Dieser Versuch, alle Emotionen aus tierethischen Begründungen auszuschließen, um möglichst rationale und nüchterne Argumente zu bilden, findet sich auch bei Tom Regan (vgl. z. B. ${ }^{2} 2004$, iii) und wurde seither von den meisten Tierethikerinnen und Tierethikern der analytischen Tradition übernommen. Dahinter steht die Idee, dass es in der Ethik primär um das Erzeugen rationaler (bzw. logischer) Konsistenz geht (vgl. z. B. Jeff McMahan 2005, 2013) und dass unmoralisches Verhalten mit irrationalem, d. h. inkonsistentem Verhalten gleichzusetzen ist.

Feministische Zugänge kritisieren diesen Rationalismus in der Orthodoxie scharf, weil diesen zufolge Emotionen und Fürsorge grundlegend dafür sind, dass Tiere so behandelt werden, wie sie behandelt werden sollten (z. B. Josephine Donovan 1990; Erin McKenna 1994). Einige Philosophinnen und Philosophen außerhalb der feministischen Tradition betonen ebenfalls die Rolle von Emotionen in der Tierethik (z. B. Ralph R. Acampora 2006; Tony Milligan 2010; Elisa Aaltola 2018). Auch in Wittgenstein'schen Zugängen, speziell jenem von Diamond (1978), gelten Emotionen wie etwa Mitleid als wesentlich für die moralische Berücksichtigung von Tieren. Mitleid sei grundlegend für unser Verständnis von Leid und 
Tod sowie dafür, was sie bedeuten und inwiefern sie für jene relevant sind, die davon betroffen sind, Tiere mit eingeschlossen. Indem sie abstrakte Argumente zur Verhinderung von Tierleid machen, gehen Singer und jene, die ihm folgen, davon aus, dass unser Verständnis für die Notwendigkeit, Leid zu verhindern, auch ohne Beteiligung von Emotionen wie Mitleid auskommt (Cora Diamond 1978, 478). Diamond markiert dies als irreführend und wirft eine „philosophische Überbetonung von Prinzipien“ vor, die Menschen von der Philosophie entfremdet und die philosophischen Argumente wenig überzeugend macht (1991 a, 57).

Selbst ein eher flüchtiger Blick auf die empirischen Belege, die auf dem Gebiet der Moralpsychologie gewonnen werden, unterstützt die Idee, dass ein übermäßiger Rationalismus ohne Rücksicht auf Emotionen in die Irre führen muss. Schon aus den Arbeiten von Antonio Damasio (2005) wissen wir, dass Emotionen nötig sind, um vom moralischen Urteil zum Handeln zu gelangen. Es gibt Hinweise, dass Emotionen unsere moralischen Urteile beeinflussen oder sogar bestimmen können (vgl. z. B. Jonathan Haidt 2003). Jesse Prinz (2006) meint z. B. unter Bezugnahme auf empirische Belege, dass Emotionen und moralische Urteile dahingehend zusammenhängen, als Emotionen typischerweise gemeinsam mit moralischen Urteilen auftreten, Emotionen oft moralische Urteile beeinflussen und Emotionen für die moralische Entwicklung wichtig sind. Er erwähnt in diesem $\mathrm{Zu}$ sammenhang das Beispiel der Psychopathie, in der schwere emotionale Defizite zu einem Fehlen von empathischem Distress, Reue und Schuldgefühlen führen. Prinz sagt sogar, dass Emotionen vielleicht notwendig für moralische Urteile sind, und zwar in dem Sinne, dass die Festlegung auf ein moralisches Urteil zugleich bedeutet, dass man zur Empfindung gewisser Emotionen in bestimmten Kontexten disponiert ist. Ein Beispiel: Beurteilt man Folterung als etwas moralisch Falsches, wird man in Situationen, in denen man Zeugin oder Zeuge einer Folterung ist, emotional nicht ungerührt bleiben.

Unabhängig davon, wie sehr man sich einem sentimentalistischen $\mathrm{Zu}-$ gang zu Fragen der Moral verpflichtet fühlt, ist es ohne Zweifel so, dass Emotionen in einem gewissen Maß Teil unseres moralischen Lebens sind. Wenn man in der Beschäftigung mit der Tierethik Emotionen gänzlich auBer Acht lässt, entgeht einem dementsprechend nicht nur ein Mittel, um Menschen zum Nachdenken zu bewegen (Emotionen können z. B. durch Videos ausgelöst werden, die bestimmte Praktiken der Tiernutzung zeigen), sondern man verkennt auch, inwiefern Menschen nicht nur rationale, sondern zugleich emotionale moralische Wesen sind. 


\subsection{Kritik am Naturalismus}

Der traditionelle MI in der Tierethik ist in dem Sinn naturalistisch, als er auf empirische Tatsachen über Tiere Bezug nimmt, um seine Argumente zu plausibilisieren. Singers utilitaristischer Zugang zur Tierethik stützt sich auf die Berücksichtigung z. B. jener Voraussetzungen, die Tiere dazu befähigen, Lust und Schmerz zu empfinden; diese Fähigkeiten erzeugen wiederum bestimmte Interessen, die es zu berücksichtigen gilt (z. B. das Interesse, nicht zu leiden). Wenn wir wissen, wie die Welt ist, so die Annahme, seien wir auch in der Lage dazu, die Gründe für unser Handeln leichter zu bestimmen, da sich diese Gründe letztlich auf natürliche Fakten beziehen würden; die angenommene Tatsache, dass sich Schmerz schlecht anfühlt und ein Interesse nach Schmerzfreiheit generiert, impliziert z. B., dass wir bestrebt sein sollten, die Menge an Schmerz in der Welt zu minimieren. Aus der Wittgenstein'schen Perspektive ist dieser Naturalismus aufgrund zweier Gründe problematisch.

Erstens, der Naturalismus nimmt an, dass wir in der Art, wie wir über Tiere denken, einen „Standpunkt des Universums“ einnehmen können (vgl. z. B. Katarzyna De Lazari-Radek/Peter Singer 2016). Dies hängt mit der Idee im traditionellen MI zusammen, von einem unparteiischen Standpunkt aus nach rein rationalen Gründen für die ethische Behandlung aller Wesen zu suchen. Genau gegenüber dieser Ansicht hat Diamond starken Vorbehalt, weil sie impliziert, dass die Gründe für die moralische Berücksichtigung von Tieren für alle (rational denkenden) Menschen Geltung besitzen, selbst für solche Menschen, denen jegliche Vorstellungskraft und jedes Mitgefühl für Tiere abgeht (Cora Diamond 1978, 479). Crary und Diamond zufolge führt der Rationalismus nicht nur zu einer wenig überzeugenden Moraltheorie, sondern der Gedanke, dass wir eine unparteiische Perspektive einnehmen könnten, sei kaum mehr als ein Wunschdenken. Die Ansätze von Diamond und Crary gehen von dem aus, was man einen „epistemischen Anthropozentrismus“ nennen kann, also einer unüberwindlichen menschlichen Perspektivität in unserer Auseinandersetzung mit der Welt und unserem Verständnis von ihr. Was die traditionelle Tierethik als einen Verlust von Objektivität ansieht, wäre aus Wittgenstein'scher Sicht sogar etwas, das man fördern und an dem man festhalten sollte (vgl. dazu Benjamin De Mesel 2018, 91-110). Anstatt des Versuchs, der Welt mit einer kalten und nüchternen wissenschaftlichen Art zu begegnen, sollte man in der Ethik auf alle Fähigkeiten zurückgreifen, die uns in unserem moralischen Leben gegeben sind, und dazu gehören z. B. auch unsere Emotionen, unsere Vorstellungskraft und unser Ergriffensein von Narrativen. 
Ein zweiter Grund, warum Crary und Diamond den Naturalismus der traditionellen Tierethik ablehnen, liegt darin, dass diese die Grundlage der Moral in den Charakteristika von Individuen findet, während die Wittgenstein'sche Perspektive diese Grundlage viel mehr in den menschlichen Praktiken annimmt. Diese Praktiken beeinflussen, wie wir die Eigenschaften der Wesen, mit denen wir interagieren, konzeptuell erfassen. Dass man einem jeweiligen Individuum, dem man begegnet, eine gewisse Aufmerksamkeit schenken muss, wird im Wittgenstein'schen Zugang nicht bestritten, aber unsere bedeutungsvermittelnden Praktiken werden hier als Grundlage thematisiert, wie wir dieses Individuum verstehen. D. h. auch, dass die im MI geforderte rationale Reflexion über tierliche Eigenschaften und deren moralische Relevanz nicht voraussetzungslos ist und es keinen unvoreingenommenen Standpunkt gibt.

Die Annahme, Praktiken seien eine wichtige Grundlage der Moral, stellt nach unserem Dafürhalten eine entscheidende Einsicht dieses Wittgenstein'schen Zugangs dar, und zwar eine Einsicht, für die der orthodoxe Zugang zur Tierethik wenig empfänglich erscheint, obwohl sie implizit in deren Argumentationen enthalten ist. Das Argument der „Grenzfälle“ als paradigmatisches Beispiel traditioneller Argumentation würde in der Tat nicht funktionieren, gäbe es nicht einen Hintergrund bestimmter Praktiken, die es stützen. Man stelle sich hierzu eine Person vor, die das Schlachten und Essen von Kühen mit dem Hinweis zu rechtfertigen sucht, dass Kühe kein Konzept des Todes haben und es ihnen deshalb egal ist, getötet oder gegessen zu werden, solange dies schmerzfrei erfolgt. Eine mögliche Antwort hierauf, die sich auf das Argument der "Grenzfälle“ bezieht, könnte so lauten, dass auch menschliche Babys kein Konzept des Todes haben, wir sie aber trotzdem nicht essen. Es wäre demnach inkonsistent, Kühe zu essen, Babys aber nicht, wenn die Rechtfertigung dafür im Verweis auf das fehlende Konzept des Todes gesucht wird. Abgesehen davon, dass das Argument vielleicht inkonsistentes Verhalten aufzeigt, kann es aber nur deshalb überzeugend für die Person in diesem Beispiel sein, weil sie dazu gebracht wird, Kühe in einem neuen Licht zu sehen, und zwar basierend auf bestehenden Praktiken im zwischenmenschlichen Bereich.

Trotz dieser Kritik am Naturalismus im traditionellen MI ist es wichtig zu betonen, dass aus Crarys und Diamonds Arbeiten nicht die vollständige Ablehnung aller Verweise auf biologische Fakten folgt. Das Wissen über biologische Fakten, die ein bestimmtes Tier betreffen, ist wichtig und kann in manchen Fällen entscheidend dafür sein, dieses Tier angemessen zu behandeln. Dies ist besonders in Fällen von Ungewissheit auffällig. Sich z. B. um einen Hund zu kümmern erfordert kaum ein Studium biologischer Fakten über das Tier, weil wir bereits ausreichend mit Hunden ver- 
traut sind, aber bei weniger vertrauten Tieren, wie z. B. Tintenfischen, kann die Berücksichtigung dieser Fakten entscheidend dafür sein, die Tiere so zu behandeln, wie sie es verdienen. Der Punkt, um den es Crary und Diamond geht, ist nicht, dass biologische Fakten außer Acht gelassen werden sollten, sondern dass diese nicht alles sind, worüber man Bescheid wissen sollte. Biologische Fakten alleine können weder moralisches Handeln noch Moraltheorien begründen, weil die Interpretation dieser Fakten je schon im Kontext bestimmter Praktiken steht. Dies bedeutet: Obwohl ein gewisser Naturalismus unvermeidlich und sogar wünschenswert ist, sollten wir eine Ethik anstreben, die empirisch informiert ist, und dabei den Versuch aufgeben, unser moralisches Leben auf das zu reduzieren, was naturwissenschaftlich beschreibbar ist.

\subsection{Kritik am Reduktionismus}

Der traditionelle MI ist reduktionistisch, weil er versucht, unser moralisches Leben auf eine Theorie zu reduzieren, die so wenige Prinzipien wie möglich postuliert und in einigen Fällen sogar monistisch ist. Der Grund dafür, warum sich so viele Ethikerinnen und Ethiker trotz seiner kontraintuitiven Implikationen z. B. vom Utilitarismus angezogen fühlen, liegt u. a. darin, dass der Utilitarismus mit wenigen Voraussetzungen auskommt. Es ist lediglich nötig, ein hedonistisches Verständnis des Guten sowie ein Aggregationsprinzip zu postulieren. Doch dies kann nur dann als Vorteil gelten, wenn man davon ausgeht, dass Moraltheorien einfach sein sollen, selbst wenn dafür in Kauf genommen werden muss, dass sie sich von unserem komplexen moralischen Leben entfernen. Es ist allerdings alles andere als klar, warum dies so sein sollte. Stattdessen könnte man die Logik dieses Arguments auch auf den Kopf stellen und argumentieren, dass der Utilitarismus genau deshalb abgelehnt werden sollte, weil er unser moralisches Leben auf eine einfache Behauptung reduziert: „Schmerz ist schlecht; Lust (Glück) ist gut; je weniger Schmerz und je mehr Lust es insgesamt gibt, desto besser“. Dementsprechend hat Mary Midgley die Kritik formuliert, dass jede Moralphilosophie, die unser moralisches Leben auf eine „einfache Formel" reduziert, letztlich als stark vereinfachend und trügerisch anzusehen ist $(1998,30)$.

Die Unterbietung der Komplexität gelebter Moral ist also ein weiterer Grund, warum der Wittgenstein'sche Zugang dem Reduktionismus des traditionellen MI kritisch gegenübersteht und stattdessen die Pluralität unseres moralischen Lebens als Ausgangspunkt ethischen Nachdenkens wählt. Obwohl Theorien im Rahmen des traditionellen MI den Vorteil lo- 
gischer Konsistenz und Einfachheit haben, lassen sie doch die Reichhaltigkeit und Komplexität unseres moralischen Lebens außer Acht. Das ist umso problematischer, wenn man berücksichtigt, dass der traditionelle MI seine Schlussfolgerungen und normative Kraft letztlich aus unserem moralischen Leben und unseren bestehenden Praktiken bezieht. So erhält das Argument gegen den Speziesismus seine Plausibilität wesentlich aus der Analogie zu Argumenten gegen Praktiken der Sklaverei, des Rassismus und des Sexismus. Die Gründe dafür, warum wir im Umgang mit einer beeinträchtigten Person, unserem Baby, unserem Nachbarn, unserer Großmutter oder einem Leichnam besonders umsichtig sind, können ganz unterschiedlich sein. Die Überbetonung von Prinzipien, logischer Konsistenz und der kleinstmöglichen Menge an normativen Bezugspunkten lässt zwangsläufig manche dieser Gründe unberücksichtigt und trägt etablierten Praktiken nicht ausreichend Rechnung. In diesem Sinne gilt, dass der traditionelle MI zwar mit logischer Schlüssigkeit und Konsistenz aufwarten kann und die Fähigkeit besitzt, das „Durcheinander“ unseres moralischen Lebens zu vereinfachen, der Wittgenstein'sche Zugang aber ein höheres Erklärungspotential hat und durch seinen pluralistischen Ansatz besser dabei helfen kann, unsere moralischen Überzeugungen in ihrer Komplexität zu verstehen.

\section{Eine Alternative zur traditionellen Tierethik?}

Die Erwägung, ob der Wittgenstein'sche Zugang tatsächlich eine solide Alternative zur traditionellen Tierethik ist, hängt davon $a b$, ob Praktiken und Paradigmen den Vorrang vor Theorie und Prinzipien haben - eine Idee, die nicht nur in der Tierethik (z. B. Elisa Aaltola 2010), sondern generell in der angewandten Ethik (z. B. Dieter Birnbacher 2003, 34-77; 2006; Tom L. Beauchamp/James F. Childress $\left.{ }^{5} 2001,384-413\right)$ und speziell in der Moralepistemologie (z. B. Richmond Campbell 2015; Susan Haack 2009; Geoffrey Sayre-McCord 1996) kritisiert wurde. Die zentrale Frage ist, ob moralische Urteile überhaupt durch eine Bezugnahme auf bereits etablierte Praktiken begründet werden können und ob eine ethische Argumentation dadurch nicht in dem verhaftet bleibt, was aktuell moralisch akzeptiert wird (und ob Ethik damit nicht zu einer Soziologie wird; Elisa Aaltola 2010, 37), oder, noch schlimmer, ob dadurch nicht das Risiko besteht, moralisch illegitime Praktiken zu reproduzieren. Ein Beispiel: Wären wir jemals auf die Idee gekommen, dass Hexenverbrennung, Sklaverei, Rassismus oder Sexismus moralisch falsch sind, wenn die einzige Quelle unserer Kritik bestehende Praktiken gewesen wären? Um auf diesen entscheidenden Punkt 
einzugehen, möchten wir drei Argumente für die Idee vorbringen, dass die Betonung der Bedeutung von Praktiken im ethischen Denken nicht zwangsläufig bedeutet, dass man damit das Potential zur Kritik an diesen Praktiken verliert.

\subsection{Theorien und Prinzipien sind nicht die einzigen Ausgangspunkte von Kritik}

Das Bedenken, dass der angenommene Vorrang von Praktiken zur Unmöglichkeit führt, diese Praktiken auch kritisieren zu können, beruht auf der Vorstellung, dass Theorien und Prinzipien (als Gegenstücke zu Praxis und Paradigmen) die einzigen Ausgangspunkte für moralische Kritik seien. Diese Annahme steht allerdings auf wackeligen Beinen. Träfe sie zu, bräuchte es die Disziplin der Moralphilosophie, um eine etablierte Praxis aus moralischen Gründen in Frage stellen zu können. Dies ist aber offenbar nicht der Fall, weil sowohl Erwachsene, die nicht mit Moralphilosophie vertraut sind, als auch Kinder durchaus die Fähigkeit besitzen, die Praktiken anderer zu kritisieren oder abzulehnen. Kuusela schreibt dementsprechend:

„[L]eading a human life involves [a pre-theoretical] understanding of a distinction between good and bad. We do not need the discipline of ethics or moralists to teach us that there is such a distinction, but it already informs our lives" (Oskari Kuusela 2018, 39).

Darüber hinaus müsste ein Gegner dieser Ansicht die Meinung vertreten, dass es in Gesellschaften ohne moralphilosophische Auseinandersetzung keine moralischen Probleme gäbe bzw. Menschen keine Möglichkeit auf moralischen Wandel und Entwicklung hätten. Solch eine Behauptung ist allerdings absurd.

\subsection{Moralische Probleme tauchen zuerst in der Praxis auf}

Moralische Probleme zeigen sich zuerst als Probleme im Zusammenhang mit einer bestehenden Praxis, nicht erst als das Resultat einer (moralphilosophischen) Theorie. Man denke z. B. an eine Tierärztin oder einen Tierarzt, die oder der einen gesunden Welpen euthanasieren soll, weil dessen Fellfarbe nicht mit den Zuchtzielen übereinstimmt (Sonja Hartnack et al. 2016). Hartnack et al. haben gezeigt, dass Tierärztinnen und Tierärzte in Österreich moralischen Stress empfinden, wenn sie einen Welpen nur aufgrund der Wünsche einer Halterin oder eines Halters euthanasieren sollen. 
Konflikte zwischen den Wünschen von Halterinnen und Haltern und deren Verantwortung für das Leben und das Wohlergehen ihres Tieres tauchen als moralische Herausforderungen auf, ohne dabei von vorherein auf Moraltheorien oder Prinzipien bezogen zu sein. Solche Konflikte wären nicht denkbar, wenn unser moralisches Leben und unsere Praktiken ohne Unterstützung von Moraltheorien oder Prinzipien immun gegenüber Zweifel und Kritik wären. Viele ethische Theorien haben sich mit der Frage beschäftigt, wie moralische Probleme entstehen, und eine wichtige Antwort darauf findet sich in unseren vorreflexiven Praktiken. So beziehen sich William David Ross' (2003) Idee von Konflikten zwischen prima facie Pflichten, Richard M. Hares (1981) Annahme von Konflikten zwischen vorreflexiven Intuitionen oder John Deweys $\left(1908,192 ;{ }^{2} 1932,164-165\right)$ Erwähnung des Scheiterns von Gewohnheiten (engl. habits) z. B. alle auf praktische Probleme, die ethische Reflexion auslösen (Herwig Grimm 2010, 141-151). Der Umstand, dass dem Handeln plötzlich die Selbstverständlichkeit fehlt, bedingt das ethische Nachdenken (Herwig Grimm 2010, 141).

Der entscheidende Punkt ist der, dass Kritikerinnen und Kritiker praxisorientierter Zugänge dazu tendieren, Praktiken als starr und nicht dynamisch anzusehen, und zwar als Handlungsfelder, in denen die Akteurinnen und Akteure ein identisches Muster an moralischen Überzeugungen und Moralverständnissen reproduzieren, die nicht miteinander kollidieren. Träfe dies zu, hätten praxisorientierte Theorien in der Tat ein schwerwiegendes Problem, weil das kritische Potential ethischen Nachdenkens verlorenginge und bestehende Praktiken nur reproduziert und gefestigt werden würden. Situationen des Zweifels und Orientierungsverlustes erzeugen den Bedarf nachzudenken, um wieder Orientierung zu gewinnen. In dieser Hinsicht können ethische Theorien und Prinzipien eine große Hilfe sein - ein Sachverhalt, der zum dritten Argument führt.

\subsection{Praxisorientierung schließt Prinzipien nicht zwangsläufig aus}

Nicht alle praxisorientierten Theorien lehnen Moraltheorie und Prinzipien ab. Eine etwas „wohlwollender“ gefasste Interpretation des Wittgenstein'schen Zugangs, als beispielsweise jene von Aaltola (2010, 31), erlaubt es, Praxis und Theorie auch vor diesem Hintergrund nicht als starke Gegensätze zu thematisieren. Es könnte argumentiert werden, dass ein instrumentalistisches Verständnis moralischer Prinzipien und Theorien wie jenes von Dewey mit einem Wittgenstein'schen Zugang zur Moral kompatibel ist. Dewey schreibt: 
"[T]he object of moral principles is to supply standpoints and methods which will enable the individual to make for himself an analysis of the elements of good and evil in the particular situation in which he finds himself. [...] A moral principle, then, is not a command to act or forbear acting in a given way: it is a tool for analyzing a special situation [...]" (John Dewey 21932, 280, Hervorhebung im Original).

Dieses instrumentalistische Verständnis erlaubt es, Theorie und Prinzipien als hochrelevant für die Ethik anzusehen, auch wenn die Praxis eine noch wichtigere Rolle spielt. Sie haben die Funktion, uns in unserem praktischen Denken anzuleiten, aber dabei dienen sie als Instrumente, nicht als Zwecke. Moraltheorie und Prinzipien können demnach als Werkzeuge verstanden werden, die uns dabei helfen, gründlicher und differenzierter nachzudenken, aber sie sind weder moralischen Praktiken vorrangig noch sollten sie als Zwecke an sich betrachtet werden. Gäbe es allerdings neben den Praktiken nicht auch noch die Fähigkeit zur ethischen Deliberation, könnten Menschen diese lebenswirklichen Probleme nicht mit ausreichender Sorgfalt bewältigen. In dieser Hinsicht sollte der Wittgenstein'sche Zugang zur Tierethik nicht als strikte Alternative zur traditionellen Tierethik aufgefasst werden. Ethische Theorien erlauben uns, besser und systematisch zu denken.

\section{Fazit}

Die Wittgenstein'sche Kritik macht die Schwierigkeiten des impliziten Rationalismus, Naturalismus und Reduktionismus traditioneller Formen des MI deutlich. Trotzdem ist es wenig plausibel, das Individuum in der Ethik gänzlich außer Acht zu lassen, weshalb sogar Crary und Diamond bis zu einem gewissen Grad eine individualistische Position einnehmen. Während daher ihre Kritik am Individualismus im MI scheitert, so hat doch der Ruf nach einer Ethik, die "menschlicher" ist, aus unserer Sicht eine starke Berechtigung: Eine Ethik nämlich, welche spezifische Eigenschaften (menschliche wie tierliche) thematisieren kann und dabei der Reichhaltigkeit und Komplexität Beachtung schenkt, die das moralische Leben charakterisieren. Dies bedeutet nicht, dass praxisorientierte Ansätze etablierte Praktiken unangetastet lassen und bestehende Ungerechtigkeiten verfestigen. Wir haben stattdessen gezeigt, dass moralische Probleme der Praxis der Theorie vorausgehen und dass Praktiken auch ohne Moraltheorie kritisiert werden können. Dies impliziert jedoch nicht, dass Moraltheorien nutzlos wären. Der Wittgenstein'sche Zugang ist daher weniger als strikte 
Alternative zur traditionellen Tierethik zu verstehen, sondern als Instrument, die orthodoxen Ansätze richtig einzuordnen und das Nachdenken über unsere moralische Verantwortung gegenüber Tieren weiterzuentwickeln. ${ }^{4}$

\section{Literatur}

Aaltola, Elisa 2010, The Anthropocentric Paradigm and the Possibility of Animal Ethics, in: Ethics and the Environment 15, 27-50; DOI:10.2979/ete.2010.15.1.27.

Aaltola, Elisa 2018, Varieties of Empathy: Moral Psychology and Animal Ethics, Lanham.

Acampora, Ralph R. 2006, Corporal Compassion: Animal Ethics and Philosophy of Body, Pittsburgh.

Beauchamp, Tom L./Childress, James F. ${ }^{52001}$, Principles of Biomedical Ethics, New York.

Birnbacher, Dieter 2003, Analytische Einführung in die Ethik, Berlin/New York.

Birnbacher, Dieter 2006, Zwischen Natur und Interesse, Frankfurt a. M.

Campbell, Richmond 2015, Moral Epistemology, in: The Stanford Encyclopedia of Philosophy, https://plato.stanford.edu/archives/win2015/entries/moral-epistemol ogy/ (abgerufen am 28.11.2019).

Crary, Alice 2010, Minding What Already Matters: A Critique of Moral Individualism, in: Philosophical Topics 38, 17-49.

Crary, Alice 2018, Ethics, in: Gruen, Lori (Hg.) 2018, Critical Terms for Animal Studies, Chicago, 154-168.

Damasio, Antonio 2005, Descartes' Error: Emotion, Reason, and the Human Brain, New York.

DeGrazia, David 1996, Taking Animals Seriously: Mental Life and Moral Status, Cambridge.

DeGrazia, David 2002, Animal Rights. A Very Short Introduction, Oxford.

De Lazari-Radek, Katarzyna/Singer, Peter 2016, The Point of View of the Universe: Sidgwick and Contemporary Ethics, New York.

De Mesel, Benjamin/Thompson, James 2015, Wittgensteinian Approaches to Moral Philosophy, in: Ethical Perspectives 22, 1-14; DOI: 10.2143/EP.22.1.3071308.

De Mesel, Benjamin 2018, The Later Wittgenstein and Moral Philosophy, Cham.

4 Acknowledgment: Diese Arbeit wurde zum Teil finanziert durch den Austrian Science Fund (FWF): Projektnummern P 31466-G32, M 2518-G32. Gedankt sei auch Katharina Dieck für ihre Unterstützung beim Literaturverzeichnis.

Der vorliegende Text ist eine Übersetzung und überarbeitete Version folgender Arbeit: Susana Monsó/Herwig Grimm 2019. 
Delon, Nicolas 2015, Against Moral Intrinsicalism, in: Aaltola, Elisa/Hadley, John (Hg.) 2015, Animal Ethics and Philosophy: Questioning the Orthodoxy, London, 31-45.

Dewey, John 1908, Ethics, New York (= Dewey, John 1976, The Middle Works 1899-1924, vol. 5 [ed. by Boydston, Jo Ann], Carbondale, IL).

Dewey, John 21932, Ethics, New York (= Dewey, John 1985, The Later Works 1925-1953, vol. 7 [ed. by Boydston, Jo Ann], Carbondale, IL).

Diamond, Cora 1978, Eating Meat and Eating People, in: Philosophy 53, 465-479.

Diamond, Cora 1991 a, The Importance of Being Human, in: Royal Institute of Philosophy Supplement 29, 35-62.

Diamond, Cora 1991 b, The Realistic Spirit. Wittgenstein, Philosophy, and the Mind, Cambridge/London.

Donovan, Josephine 1990, Animal Rights and Feminist Theory, in: Signs 15, 350-375.

Francione, Gary 1995, Animals, Property \& The Law, Philadelphia.

Gaita, Raimond 2005, The Philosopher's Dog: Friendships with Animals, New York.

Grimm, Herwig 2010, Das moralphilosophische Experiment. John Deweys Methode empirischer Untersuchungen als Modell der problem- und anwendungsorientierten Tierethik, Tübingen.

Gruen, Lori 2014, Dignity, Captivity, and an Ethics of Sight, in: Gruen, Lori (Hg.) 2014, The Ethics of Captivity, Oxford, 231-247.

Haack, Susan 2009, Evidence and Inquiry: A Pragmatist Reconstruction of Epistemology, New York.

Haidt, Jonathan 2003, The Moral Emotions, in: Davidson, Richard J./Scherer, Klaus R./Goldsmith, H. Hill (Hg.) 2003, Handbook of Affective Sciences, Oxford/New York, 852-870.

Hare, Richard M. 1981, Moral Thinking: Its Levels, Method and Point, Oxford.

Hartnack, Sonja/Springer, Svenja/Pittavino, Marta/Grimm, Herwig 2016, Attitudes of Austrian veterinarians towards euthanasia in small animal practice: Impacts of age and gender on views on euthanasia, in: BMC Veterinary Research 12, 1-14; DOI: 10.1186/s12917-016-0649-0.

Horta, Oscar 2014, The Scope of the Argument from Species Overlap, in: Journal of Applied Philosophy 31, 142-154.

Hursthouse, Rosalind 2006, Applying Virtue Ethics to our Treatment of the Other Animals, in: Welchman, Jennifer (Hg.) 2006, The Practice of Virtue: Classic and Contemporary Readings in Virtue Ethics, Indianapolis, 136-155.

Hursthouse, Rosalind 2011, Virtue Ethics and the Treatment of Animals, in: Beauchamp, Tom L./Frey, R. G. (Hg.) 2011, The Oxford Handbook of Animal Ethics, New York; DOI: 10.1093/oxfordhb/9780195371963.013.0005.

Kuusela, Oskari 2018, Ethics and Philosophical Clarification, in: Agam-Segal, Reshef/Dain, Edmund (Hg.) 2018, Wittgenstein's Moral Thought, New York, 37-65. 
May, Todd 2014, Moral Individualism, Moral Relationalism, and Obligations to Non-Human Animals, in: Journal of Applied Philosophy 31, 155-168.

McKenna, Erin 1994, Feminism and Vegetarianism: A Critique of Peter Singer, in: Philosophy in the Contemporary World 1, 28-35.

McMahan, Jeff 2005, Our Fellow Creatures, in: The Journal of Ethics 9, 353-380; DOI: $10.1007 /$ s10892-005-3512-2.

McMahan, Jeff 2013, Infanticide and Moral Consistency, in: Journal of Medical Ethics 39, 273-280; DOI:10.1136/medethics-2012-100988.

McReynolds, Phillip 2004, Overlapping Horizons of Meaning: A Deweyan Approach to the Moral Standing of Nonhuman Animals, in: McKenna, Erin/Light, Andrew (Hg.) 2004, Animal Pragmatism. Rethinking Human-Nonhuman Relationships, Bloomington, 63-85.

Midgley, Mary 1998, Animals and Why They Matter, Athens.

Milligan, Tony 2010, Beyond Animal Rights: Food, Pets and Ethics, London/New York.

Monsó, Susana/Grimm, Herwig 2019, An Alternative to the Orthodoxy in Animal Ethics? Limits and Merits of the Wittgensteinian Critique of Moral Individualism, in: Animals 9 (12), art. 1057; DOI:10.3390/ani9121057.

Nussbaum, Martha C. 2007, Frontiers of Justice: Disability, Nationality, Species Membership, Cambridge.

Palmer, Clare 2010, Animal Ethics in Context, New York.

Pluhar, Evelyn B. 1995, Beyond Prejudice: The Moral Significance of Human and Nonhuman Animals, Durham.

Prinz, Jesse J. 2006, The Emotional Basis of Moral Judgements, in: Philosophical Explorations 9, 29-43; DOI: 10.1080/1386979050049246.

Rachels, James 1999, Created from Animals: The Moral Implications of Darwinism, New York.

Regan, Tom 22004, The Case for Animal Rights, Berkeley.

Rollin, Bernard 22017, The Unheeded Cry: Animal Consciousness, Animal Pain, and Science, Columbia.

Ross, William David 2003, The Right and the Good, New York.

Ryder, Richard 1971, Experimenting on Animals, in: Godlovitch, Stanley/ Godlovitch, Rosalind/Harris, John (Hg.) 1971, Animals, Men, and Morals, New York, 41-82.

Sayre-McCord, Geoffrey 1996, Coherentist Epistemology and Moral Theory, in: Sinott-Armstrong, Walter/Timmons, Mark (Hg.) 1996, Moral Knowledge: New Readings in Moral Epistemology, New York, 137-189.

Singer, Peter ${ }^{4} 2009$, Animal Liberation. The Definitive Classic of the Animal Movement, New York.

Varner, Gary E. 2012, Personhood, Ethics, and Animal Cognition: Situating Animals in Hare's Two Level Utilitarianism, New York. 
